\title{
Stress in cynomolgus monkeys (Macaca fascicularis) subjected to long-distance transport and simulated transport housing conditions
}

Authors: A. L. Fernström ${ }^{\text {ab }}$; W. Sutian ${ }^{\text {ac }}$; K. Westlund ${ }^{\text {d }}$ T. Nilsson ${ }^{\text {a }}$ H. -E. Carlsson ${ }^{\mathrm{a}}$; Y. Paramastric; J. Pamungkas $^{\text {c}}$; D. Sajuthi ${ }^{\text {c }}$ S. J. Schapiro ${ }^{\text {e; J. Hau }}{ }^{\text {ab }}$

\author{
Affiliations: ${ }^{a}$ Department of Neuroscience, Comparative Medicine, Uppsala University, \\ Uppsala, Sweden \\ b Department of Experimental Medicine, University of Copenhagen and State \\ Hospital, The Panum Institute, 2200 N Copenhagen, Denmark \\ ${ }^{c}$ Primate Research Centre, Bogor Agricultural University, Bogor, 16151, Indonesia \\ d The Swedish Institute for Infectious Disease Control, Stockholm, Sweden \\ ${ }^{\mathrm{e}}$ The University of Texas M. D. Anderson Cancer Center, Bastrop, TX, USA
}

\begin{abstract}
The stress associated with transportation of non-human primates used in scientific research is an important but almost unexplored part of laboratory animal husbandry. The procedures and routines concerning transport are not only important for the animals' physical health but also for their mental health as well. The transport stress in cynomolgus monkeys (Macaca fascicularis) was studied in two experiments. In Experiment 1, 25 adult female cynomolgus monkeys were divided into five groups of five animals each that received different diets during the transport phase of the experiment. All animals were transported in conventional single animal transport cages with no visual or tactile contact with conspecifics. The animals were transported by lorry for $24 \mathrm{~h}$ at ambient temperatures ranging between $20^{\circ} \mathrm{C}$ and $35^{\circ} \mathrm{C}$. Urine produced before, during and after transport was collected and analysed for cortisol by enzyme-linked immunosorbent assay (ELISA).
\end{abstract}

All monkeys exhibited a significant increase in cortisol excretion per time unit during the transport and on the first day following transport. Although anecdotal reports concerning diet during transport, including the provision of fruits and/or a tranquiliser, was thought likely to influence stress responses, these were not corrobated by the present study. In Experiment 2, behavioural data were collected from 18 cynomolgus macaques before and after transfer from group cages to either single or pair housing, and also before and after a simulated transport, in which the animals were housed in transport cages. The single housed monkeys were confined to single transport cages and the pair housed monkeys were kept in their pairs in double size cages.

Both pair housed and singly housed monkeys showed clear behavioural signs of stress soon after their transfer out of their group cages. However, stress-associated behaviours were more prevalent in singly housed animals than in pair housed animals, and these behaviours persisted 
for a longer time after the simulated transport housing event than in the pair housed monkeys. Our data confirm that the transport of cynomolgus monkeys is stressful and suggest that it would be beneficial for the cynomolgus monkeys to be housed and transported in compatible pairs from the time they leave their group cages at the source country breeding facility until they arrive at their final laboratory destination in the country of use.

Keywords: Behaviour; macaque; pair-housing; primate; urinary cortisol; welfare 